\title{
COMMISSION 42: CLOSE BINARY STARS (ÉTOILES DOUBLES SERRÉES)
}

Report of Meetings, 15, 17, 18 and 21 August 1979

PRESIDENT: G. Larsson-Leander SECRETARY: C.D. Scarfe

As scheduled, Commission 42 convened for two business meetings, on August 15 and 21 , and two scientific meetings, on August 17 and 18. The theme of the first scientific meeting was "Recent Progress in Close Binary Studies". The programme for this meeting grew to such extent that the allotted time was unsufficient, and the meeting was therefore continued on August 21, after the end of the business session. The second scientific meeting, August 18, was a Joint Meeting together with Commissions 10, 27, 40, 44 and 48 on the topic "Close Binaries and Stellar Activity". In addition, there were three informal meetings, viz. August 17 a meeting on VV Cep arranged together with Commission 29, and August 18 and 22 joint meetings with Commissions 29, 40 and 48 for the purpose of discussing SS 433. Reports from all these meetings are given below.

\section{Business Meeting I - August 15, 1979}

Condensed minutes of this meeting, which started at 14.15 and ended at 15.45 , are as follows:

1. After having adopted the agenda, as listed on the black board by the President, the Commission elected C.D. Scarfe as Secretary for the meetings. He also agreed to act as correspondent to the General Assembly journal Meteor.

2. The President asked for a moment of silence in memory of the three Commission members, J.F. Heard, S.-S. Huang and C. Popovici, who had died since the last IAU General Assembly.

3. The President announced that C.M. Huffer and H. Kristenson wish to withdraw from the Commission, as they are no longer active in the field. During the past triennium K.G. Kron, B.F. Marino, E.F. Milone and R.M. Williamon were co-opted as Commission members. With these changes, the Commission counts 130 members. The President read a list of 63 new applications for membership, divided as follows: 24 individual requests from present IAU members, 3 individual requests and 36 recommendations from National Committees concerning persons now proposed as new members of the IAU. The Organizing Committee will consider all these names, and the Committee was authorized by the Commission to decide upon the question of admittance and to report on the outcome at the second business meeting. The President pointed out that most of the applications came from persons highly qualified in the field of close binaries and that the large majority were likely to be accepted. One further member, T.H. Morgan, was proposed by Kondo.

4. The President listed the members of the present Organizing Committee with their respective years of service and said that as expected, B. Warner, the VicePresident, would be the next President. The Commission is proposing A. Batten as next Vice-President, with $\mathrm{J}$. Smak as an alternative. M. Plavec and T. Herczeg, who had served for a long time, asked to be allowed to stand down from the Organizing Committee. The names of L.B. Lucy and R.H. Koch were proposed as replacements. Koch agreed and Leung was asked to contact Lucy, who was absent. As expected, a positive answer was received from Lucy. 
5. The President read a letter from K. Gyldenkerne, Chairman of the Coordinated Programs Committee, who wishes to resign from the Committee and recommends its abolition. This view was supported by Andersen and Merrill. The Committee provided encouragement and advice but was able to offer little else, according to Kondo and Bolton. Wood pointed out that nowadays communication between those participating in such programmes is better than formerly, and Herczeg suggested that the organizing Committee could take over the responsibility of organizing co-ordinated programmes. Merrill stressed the importance of collecting and publishing the results of campaigns. The Commission agreed to abolish the Coordinated Programs Committee.

6. The Committee for Extraterrestrial Observations was discussed. The Chairman of this Committee, Y. Kondo, feels that it has fulfilled its purpose, but he is willing to continue as a clearing house of information and watchdog. It is quite obvious that the X-ray binary campaigns have been successful, e.g. Cyg X-1 (Bolton) and X Per (de Loore). The newsletter on X-ray binaries has been continued till 1978. It was agreed to keep the Committee but no longer to publish the newsletter.

7. Kondo spoke on the use of HEAO-2 (Einstein) and IUE satellites. HEAO-2 is expected to last one more year, so those who wish to propose observations should at once contact Dr. Fred Seward, Center for Astrophysics, 60 Garden Str., Cambridge, MA 02138 , U.S.A. Mattei pointed out that many optically variable objects observed by HEAO-2 are being followed by AAVSO members simultaneously. IUE is geosynchronous; $2 / 3$ of its time is controlled by the U.S. and 1/3 by Europeans. It has high and low resolution spectrometers. Prospective observers should contact Dr. Don West, Code 685, Goddard Space Flight Center, Greenbelt, MD 20771, U.S.A. Kondo supplies an IUE newsletter. The IUE lifetime should be 3 to 5 years.

8. Koch, reporting on the work on the new Finding List, said that this would soon be available with information on 3555 stars in our Galaxy. For over 100 stars the most recent photometric analyses are based on visual or photographic estimates. Herczeg suggested that publishing a list of these would inspire observers to obtain more accurate data. Merrill surmised that many may be of long period and hard to observe.

9. The President read a letter from I.-S. Nha, Yonsei University, Seoul, reporting that he is preparing an Atlas of O-C.Diagrams of Eclipsing Binaries. The work will take 2 or 3 more years, and as a by-product times of minima are collected and stored on computer cards. A data centre for the times of minima will thus exist in Seoul. Hall mentioned that such data are also collected in Cracow, by Kreiner, who will soon publish lists of times of minima for all B Lyraes, to be followed by similar lists for RS CVn's, W UMa's and eventually Algols. Wood pointed out that the duplication of effort ensures that data will not be lost in any disaster.

10. Following a proposal by Merrill, it was decided to send a card with greetings to Dr. D.J.K. O'Connell, past-President of the Commission; members were invited to sign this card.

The meeting then ajourned.

\section{Business Meeting II - August 21, 1979}

The meeting started at 9.15 and ended at 9.40. Condensed minutes are as follows:

1. The President gave a brief report from the Commission 5 Joint Meeting on Nomenclature, on August 14. The main decisions of interest to Commission 42 are:

1. Authors are strongly recommended to identify stars, whenever possible, in at least two ways (e.g. by name and by catalogue number).

2. When abbreviations for not widely known catalogues and lists of stars are used, authors should clearly indicate the meaning by giving proper references. 
2. The President described the work on the Commission Report, printed in Vol. A of the IAU Transactions. Preprints of the Report had been circulated to Commission members, but as the Report was prepared in camera-ready form there was no opportunity to correct mistakes. The President extended his thanks to the colleagues who wrote parts of the Report and to all. Commission members who furnished information.

3. With reference to decisions mentioned under item 3 of the minutes of Business Meeting I, the President stated that 51 new members had been admitted into the Commission. The new members, the names of which were included in the membership list posted on the advertisement board, are as follows: C.T. Bolton, B.B. Bookmyer, B.W. Bopp, A.N. Bunner, J.V. Clausen, G.W. Collins, J.-P. De Grève, M.J.H. de Groot, H.W. Duerbeck, J.A. Eaton, B.P. Flannery, M. Friedjung, C.D. Garmany, D.M. Gibson, E.F. Guinan, G. Hammerschlag-Hensberge, D.P. Hube, P.C. Joss, J.M. Kreiner, M.I. Kumsiashvily, Z. Kviz, D.Q. Lamb, J.A. Mattei, E. Meyer-Hofmeister, T.H. Morgan, K. Nariai, R.E. Nather, J.P. Oliver, G.J. Peters, V. Piirola, H. Ritter, E.L. Robinson, M. Rodonò, I.W. Roxburgh, N.B. Sanwal, A. Sanyal, H.J. Schober, N.I. Shakura, G. Shaviv, F. Shu, R.C. Smith, J.-E. Solheim, W.M. Sparks, D. Sugimoto, P. Szkody, J. van Paradijs, O. Vilhu, W.S.G. Walker, R.F. Webbink, E.J. Weiler, A. Yamasaki.

4. The President agreed to edit the Bibliography and Program Notes on Close Binaries for another 3 years, and indicated on a query by Merrill that his university could continue to absorb the cost for doing so. Hall suggested that the Bibliography be sent to Astronomy and Astrophysics Abstracts, and asked how widely it was disseminated to non-members. The President replied that its existence is mentioned in all Commission Reports and that it is sent to some 70 astronomers, who are not members but have asked for it.

5. Recent and future meetings were discussed. Among recent meetings the President mentioned the Rochester Colloquium on "White Dwarf's and Variable Degenerate Stars", which was co-sponsored by the Commission, and our own Symposium, "Close Binary Stars", in Toronto. He also mentioned that the Commission is co-sponsoring IAU Symposium No. 93, "Fundamental Problems in the Theory of Stellar Evolution", in Kyoto, July 1980, and a proposed Symposium on "Pulsars" in Bonn, August or September 1980. As to the future, Leung suggested a Symposium in 1981 in Hong Kong on "Contact and Related Objects", and Friedjung proposed a meeting on "Symbiotic Stars" before the next General Assembly. Both suggestions were referred to the Organizing Committee for consideration. Also, it was mentioned that Commission 26 is planning a Colloquium in Flagstaff in 1981, which may be of interest to Commission 42, and there will probably be another Bamberg Colloquium.

6. Hall reported briefly on the Second RS CVn Workshop, held in Montreal on August 13, 1979. He asked for new names, addresses and telephone numbers for the RS CVn hot line. Reports will continue to be handled by Hall and Catalano. Catalano will be writing a review paper on BY Dra stars, and Hall one on photometric aspects of RS CVn stars. Preprints will be welcomed by both. Kreiner will publish eclipse ephemerides for RS CVn stars in a forthcoming IBVS. The IAU Cire will list new RS CVn stars. The Working Group on RS CVn Binaries will continue its work.

7. The President said that the General Secretary had stressed the importance of having some written Working Rules for the various Commissions. The preparation of the rules would be a task for the new President and the Organizing Committee.

8. The President asked for proposals for new co-ordinated programmes. There were none. Those who wish to propose such programes in the next 3 years are adviced to contact the new President, B. Warner.

The meeting then ajourned. 


\section{Scientific Meeting I - August 17 and 21}

The first scientific meeting, on the theme "Recent Progress in Close Binary Studies", was started with two sessions on August 17 and continued on August 21, after the end of Business Meeting II. The following papers were presented:

J. Merrill - Some aids in the solution of light curves of eclipsing binaries with elliptical orbits.

D.M. Popper - A review of stellar masses.

J. Andersen, J.V. Clausen and B. Nordström - Recent progress in the Copenhagen programme of absolute stellar dimensions.

J. Andersen, J.V. Clausen, B. Nordström and B. Reipurth - uvby light curves of the unique RS CVn binary TY Pyx.

K.-C. Leung - Early-type semidetached and marginal semidetached systems.

D.Ya. Martynov - RX Cas: Preliminary report on the results of the co-ordinated campaign of observations (presented by A.M. Cherepashchuk).

D. Chochol - The close binary SZ Cam - a semidetached system.

H. Al-Naimiy - Photometric observations and light-curve analysis of the peculiar system ER Vul.

A.R. Sadik - Light and colour curves of UV PSC.

S.A. Naftilan - The complicated close binary CG Cyg.

G.J. Peters - Ha observations of Algols.

C.T. Bolton - The orbit and mass flow in the BN star HDE 235679.

M.G. Fracastoro - An analysis of the Barr effect.

$K$. Walter - Oblique axes of rotation in close binaries and the photometric proof of precessional motions.

T. Mazeh - On the tidal evolution of close triple systems.

I.S. McLean - Optical polarimetry of close binary systems.

V. Piirola - Polarimetry of $\mathrm{CH}$ Cyg.

G.T. Bath - Accretion powers the brightest stars.

G. Shaviv, M. Shara and D. Prialnic - Generalized scheme for novae explosions.

I. Nevo - The eruptions of the cataclysmic variables and the mass ratios of their components (presented by T. Mazeh).

K. Nomoto and K. Nariai - Recurrence of nova explosions.

M. Fujimoto - Composition of grains and theory of nova explosions.

J. Ziólkowski and B. Paczynski - A binary model and disc parameters for Cyg X-2.

C.C. Wu - IUE observations of the $\mathrm{X}$-ray nova Cen $\mathrm{X}-4$.

\section{Scientific Meeting II - August 18}

The second scientific meeting was a Joint Meeting together with Commissions 10 , 27, 40, 44 and 48 on the topic "Close Binaries and Stellar Activity". The proposal for this meeting originated from D.S. Hall, and it was planned by an organizing committee, as follows: G. Larsson-Leander (Com. 42, Chairman), J.L. Linsky (Com. 10), D.S. Hall (Com. 27 and 42), R.M. Hjellming (Com. 40), Y. Kondo (Com. 44).

The meeting occupied two sessions, the first chaired by Larsson-Leander and the second by Hall. After a short introduction by Hall, the following review talks were given:

B.W. Bopp - BY Dra and RS CVn stars: The discoveries of classical photometry and spectroscopy.

Y. Kondo - A review of recent ultraviolet observations of close binaries from space.

J.H. Swank - X-ray observations of stellar coronae and winds.

R.M. Hjellming - Radio aspects of stellar activity in close binaries.

I.S. McLean - Polarimetry of emission lines as a probe of stellar winds.

J.L. Linsky - On the differences at chromospheric levels between RS CVn-type binaries, active and quiet single stars, and active and quiet regions in the sun.

J.L. Modisette - Mass transfer between binary stars. 
The Introduction and shortened versions or abstracts of the review papers are published in Vol. 5 of the Highlights of Astronomy.

\section{Informal Meeting on VV Cephei - August 17}

This informal meeting, together with Commission 29, had been proposed by $R$. Faraggiana and was organized by her. K.0. Wright was Chairman, and some 25 persons especially interested in VV Cep participated.

The photometric data from the recent eclipse were first discussed. S. Kawabata presented a review of work done in Japan, including light curve analysis. Wright described his spectroscopic observations, now covering a whole period in high dispersion. Ultra-violet spectroscopy was reported by W. Hagen and by Faraggiana. I.S. McLean reviewed polarization observations. Kawabata described $\mathrm{H \alpha}$ observations and outlined an envelope model, worked out by M. Saito et al. A.P. Bernat made a comparison of VV Cep with 31 and 32 Cyg.

\section{Informal Meetings on SS 433 - August 18 and 22}

These two informal meetings for discussing the extraordinary object SS 433 were held jointly with Commissions 29,40 and 48 . They were organized by A. Mammano, who also served as Chairman. About 200 persons attended, and the following 20 short contributions were presented:

B. Sherwood - Distance to SS 433.

K.W. Weiler and N. Panagia - Plerionic (Crab-like) structure in W50.

R. Schilizzi - VLBI at $6 \mathrm{~cm}$ with Westerbork, Effelsberg and Onsala telescopes.

B. Geldzahler - VLBI detailing structure of a few milliarcsec (jet?).

W.S. Gilmore and E.R. Seaquist - High resolution band $20 \mathrm{~cm}$ synthes is map.

Yu.N. Parijskij - Multifrequency simultaneous monitoring and resolution of the "jet" with the $600 \mathrm{~m}$ telescope.

W.M. Goss - An H I absorption profile line with Westerbork telescope.

B. Zealey - Discovery of optical counterpart of SNR W50, centred on SS 433 .

E. Becklin and G. Wynn-Williams - Dramatic IR changes observed at Mauna Kea.

J. Bally - Brackett alfa to Brackett beta line ratios from central lines.

B. Margon - Implementation to the kinematic model for the moving features.

C. Zirmani - Optical spectra showing structures.

A. Mammano - Multiple branches and velocity changes in optical spectra up to $11000 \AA$.

B. Cosmovici and F. Strafella - Profile changes of stationary O I 8446 (presented by A. Mammano).

D. Crampton and J.B. Hutchings - Periodic variations of positions of central emission and absorption lines (13 days period).

J. Kemp - Photoelectric observations concerning the 13-d period (presented by G.W. Collins).

G.W. Collins and G.H. Newsom - On the nature of SS 433 ; a binary model.

I.S. Shklovskij - Jets produced by a very fast spinning NS $(0.001 \mathrm{~s})$ in a postsupernova binary system.

V. Icke - Clouds ejected by a disk-black-hole system.

M. Milgrom - Thomson scattering model for the secondary branches (presented by A. Mammano). 